\title{
A CIRCULATION HYPOTHESIS OF SPIRAL GALAXIES
}

\author{
CHEN LINFEI \\ Yunnan Observatory, the Chinese Academy of Science, China
}

\section{Circulation Hypothesis}

A circulation hypothesis of spiral galaxies is proposed here to explain their spiral structure: the spiral arm material is going to the galactic center along a spiral orbit and then ejects from the galactic center in the form of two-way jets in opposite directions and finally, the ejected jets return to the galaxy in the form of spiral arms again, thereby forming a closed circulatory system.

\section{Examination and Application}

It could be inferred from the circulation hypothesis that a spiral galaxy should have the two-armed spiral pattern. Observations have shown that most spiral galaxies have the two-armed spiral pattern (the reason why a few spiral galaxies haven't the two-armed spiral pattern needs to be further discussed).

While the electing directions of the jets don't coincide with the galactic symmetric plane, there must be a warp in the spiral arms regarded as the backflows of the jets since the two-way jets locate in the opposite hemispheres respectively. Observations have shown that the warp of the gaseous disk of disk-shaped galaxies is a general phenomenon.

It is known from the circulation hypothesis that the velocity of the spiral arm material should have an inward radial component and the bar material of a barred spiral galaxy should have an inward flow along the bar. Researches on the Galaxy have shown that the velocity of the early type stars near the sun has a large-scale inward radial component $v, v=-25 \pm 6$ $\mathrm{km} \mathrm{s}^{-1}$ (Zhao 1984); It has also been shown from observations that the bar material has indeed an inward flow along the bar (Carranza \& Aguero 1989). 
Now let's explain the flat rotation curve of the gases of the spiral arms in the prerequisite of the circulation hypothesis. It may be caused by the following three elements: (1) magnetic fields in the spiral arms, (2) the magnetic Reynolds number $R_{M}$ of the gases of the spiral arms far greater than 1 and (3) the specific value $k$ of the magnetic energy density of the magnetic field of the spiral arms to their thermal energy density equal to 3.3. So the magnetic field is certainly frozen in the spiral arm plasma and restricts the motion of spiral arm material, so that the magnetic field of the spiral arms and the spiral arm material controlled by the magnetic field could only be treated as a whole. it is obvious that the magnitude of the going velocity of the spiral arms should be the same everywhere, thereby inferring easily the flat rotation curve of the gases of spiral arms.

The bar structure in barred spiral galaxies may be explained by analyzing the influence of action upon the spiral arms also in the prerequisite of the circulation hypothesis. For convenience, a spiral arm is assumed as a logarithmic spiral line with a spiral angle $\alpha, \rho=\rho_{0} e^{a \theta}$ (where $a=t g \alpha$. The spiral arm material in with a galactic centric distance $r$ is acted upon in the normal direction by two forces: One is the normal component of the gravitation towards the galactic center $F_{1}=G m M(r) \cos \alpha / r^{2}$ (the formula is an approximate one, where $M(r)$ is the mass of the material within radius $r$ ) and the other is the outward normal centrifugal force $F_{2}=m v^{2} \cos \alpha / r$. The resultant force acting on the spiral arm in the outward normal direction is $F=F_{2}-F_{1}$. There might be one position $r=r_{0}$ where $F_{1}=F_{2}$. So $F$ is greater than zero in the region $r>r_{0}$, and the direction of stress of one spiral arm is just opposite to that of the other spiral arm, i.e., the resultant forces of two spiral arms in the region $r>r_{0}$ just from a pair of shearing forces. Acted by the shearing forces, the bar structure could form in some spiral galaxies.

According to the circulation hypothesis, we may foretell that there should be the two-way jets at the center of every spiral galaxy, and there should be some link between the jet material and the spiral arms.

The author considers that the cooling flows going to the galactic center in some giant elliptical galaxies may also be the backflows of the observed two-way jets and that the disk-like material near young stars is going to the centric region along a spiral orbit, and the disk-like structure itself is also the backflow of the observed two-way jets.

\section{References}

Carranza, G.J., Aguero, E.L. (1989) Ap $\mathcal{G} S S, \mathbf{1 5 2}, 279$

Zhao, J.L. (1984), Science of China, Series A, 7, 647 